\title{
How RNs rescue patients: a qualitative study of RNs' perceived involvement in rapid response teams
}

\author{
Linda Searle Leach, ${ }^{1}$ Ann Mayo, ${ }^{2}$ Maria O'Rourke ${ }^{3}$
}

${ }^{1}$ UCLA School of Nursing, Los Angeles, California, USA

${ }^{2}$ Philip Y Hahn School of Nursing and Health Science, University of San Diego, San Diego, California, USA

${ }^{3}$ UCSF \& UCLA School of Nursing, Maria W O'Rourke, Larkspur, California, USA

\section{Correspondence to} Dr Linda Searle Leach, UCLA School of Nursing, 700 Tiverton Avenue Factor Bldg 3-234, Los Angeles, CA 90095-6917, USA: lleach@sonnet.ucla.edu

Accepted 22 August 2009 Published Online First 8 April 2010
ABSTRACT

Background Patients are admitted to hospitals everyday with clinical conditions that can change dynamically resulting in adverse outcomes. The rapid response team (RRT) intervention is a formalised resource to respond to the nurse's concerns about such patients before code arrest occurs. Registered nurses (RNs) are in a position to recognise critical changes and to rescue patients at the most opportune moments, but little is known about how RNs rescue patients using these increasingly popular teams. Our aims were to investigate how RNs rescue patients in hospitals where RRTs are in place.

Methods Fifty participants involved with RRTs participated in semistructured individual interviews in six California hospitals. Data were analysed using coding and constant comparison methods.

Results Overall, RNs view RRTs as a helpful and effective safety intervention. RRT RNs and bedside RNs support one another in a synergistic way to prevent adverse patient events during the rescuing process. However, traditional hierarchies and relationships with physicians and supervisors impede some components of RN decision-making during rescuing.

Conclusions RNs find the RRT supportive when their patient is at risk or care needs are changing. They benefit from a formalised mechanism that enables immediate access to resources. RRT RNs in this study applied their expertise with critically ill patients to rescue medical and surgical patients. The RRT RN and bedside RNs' interaction influenced the rescuing process. Their role synergy was a value-added contribution to preventing adverse events and to improving patient safety by RNs.

Healthcare quality and safety efforts in the US led to a programme of initiatives by the Institute for Healthcare Improvement to significantly reduce patient mortality. ${ }^{1}$ These initiatives (Saving $100 \mathrm{~K}$ Lives Campaign) included rapid response teams (RRTs) which were developed in response to a high degree of variation in death rates among hospitals. ${ }^{2}$ Designed to rescue patients by preventing non-ICU code arrests and reducing mortality in hospitalised patients, the RRT intervention is a formalised resource to respond to the bedside RNs' concerns about a patient before a code arrest occurs. Despite enthusiasm about these teams, the evidence about their impact on reducing patient mortality has been somewhat controversial. Quality-improvement data and other reports indicate a contribution to increased numbers of saved lives. ${ }^{3-6}$ In contrast, other studies have revealed inconclusive evidence about reduced mortality or fewer code arrests over time, and although life-saving in many instances, RRTs do not prevent all avoidable cardiac arrests. ${ }^{7-10}$
Registered nurses (RNs) are in a position to recognise subtle and critical changes in order to rescue patients and make decisions to intervene at the most opportune moments to prevent adverse outcomes. Rescuing involves recognition of diagnostic cues and patient risks, intensive resource application and prevention of life-threatening events. ${ }^{2}$ Rescuing is a component of the RN role. $\mathrm{RNs}$ are authorised to and responsible for observing the patient condition, determining abnormal signs and symptoms, and making decisions to act in the best interests of the patient. Rescuing is a process of care that is nurse-sensitive, meaning it is influenced by the presence and action of the $\mathrm{RN},{ }^{11}$ and includes monitoring, surveillance, defined as the 'purposeful and ongoing acquisition, interpretation, and synthesis of patient data for clinical decisionmaking, ${ }^{12}$ vigilance, and acting to prevent an adverse outcome. Nurses intervene to manage the patient condition, appropriately reporting, referring or initiating emergency procedures to 'rescue' patients. They avert crises through rescuing, which is often referred to as the 'near miss' phenomenon where adverse outcomes are avoided due to the actions of the RN. Nurse surveillance and rescue efforts affect mortality and recovery. ${ }^{13-18}$ However, since clinical diagnostic cues are not always distinct when they first emerge, and initial cues may be subtle, disparate and unrelated, there are times when rescuing requires expert clinical decisionmaking to prevent an adverse patient outcome. Although the RN role is important to patient safety, the prevention of adverse events and rescuing, there was no research about the $\mathrm{RN}$ role in rescuing in RRTs from the perspective of those involved. Therefore, the purpose of this study was to explore and describe the RNs' role in rescuing patients in the context of having participated in an RRT intervention.

\section{METHODS}

The RRT as a care process was examined to describe the role that RNs play in assessment of the patient condition, decision-making and exercising vigilance. A qualitative approach was used to understand the processes involved in making the decision to call the RRT and the roles nurses played in the process. Sites included six acute care hospitals in northern California that had implemented RRTs and represented different classifications of organisations: non-profit community, magnet-designated, public, academic, for-profit community and integrated delivery system hospitals. Institution Review Board approval was obtained at all sites. Purposeful sampling was utilised to select key staff informants. Data were collected through individual 
semistructured interviews for approximately $1 \mathrm{~h}$ from 50 participants: 14 bedside staff RNs who had called RRTs, 16 RRT staff RNs, two respiratory therapists who had responded to RRTs and 18 nurse supervisors who had observed RRTs. All volunteered to participate and signed a written consent. Interviews were digitally recorded, transcribed verbatim without any identifying data and reviewed for accuracy.

A grounded theory approach was used for data analysis. Investigators individually read all transcripts line by line and used open coding. Further analysis involved constantly comparing and contrasting these coded data to develop broader categories and themes. The final procedures utilised axial coding to identify relationships and interconnections between codes while considering causal conditions and consequences. Respondent validation was addressed in a meeting of the participants where the research findings were presented, feedback was obtained and interpretation of the data was assessed for accuracy from the perspective of the participants.

\section{RESULTS}

\section{Rescuing process and RN decision-making}

The bedside RNs' role in rescuing patients involved calling the RRT. Many different patient changes acted as cues for the bedside RNs to decide to call the RRT. Bedside RNs assessed patient status using traditional physiological cues such as changes in the patient's heart rate or blood pressure or an alteration in the level of consciousness and/or orientation. Bedside RNs also assessed patient status by applying their knowledge of the patient to identify less objective cues, such as 'Something is not right or I am concerned.' RNs used knowledge of their hospital protocol for calling the RRT using selected vital sign criteria determined by each setting. Each site had slight variations in such parameters, but all sites used respiratory rate, heart rate, systolic blood pressure, level of consciousness and being worried. ${ }^{19}{ }^{20}$ Based on one, several or all of these cues, RNs made a thought decision to call the RRT. 'Thought decisions' are a cognitive activity to determine or think through what action to take, characterised by the $\mathrm{RN}$ using assessment data, their interpretation of that data and their clinical experience to think about the appropriate course of action. When making such thought decisions as, 'I think I will call the RRT,' their rationale was to get support and affirmation that there was a need for help; to get additional resources (ie, equipment, respiratory therapy, physician intervention); and/or to get urgent assistance to prevent a code arrest. Once the thought decision was made, some RNs sought validation by consulting with other RNs, the unit nurse manager or a clinical nurse specialist before enacting the decision to call the RRT. This reflective evaluation was reported particularly when the nurse was calling an RRT for the first time. Then, the RN made an 'enactment decision,' which was to choose the action to take, to call the RRT or not. An enactment decision was a cognitive activity nurses used to resolve the situation or problem at hand, encompassing reflective thinking targeted to future actions that would be needed. ${ }^{21}$ The nurse's cognitive work involved selecting from a choice of actions. Once the enacted decision to call the RRTwas made, the RN then made the 'operational decision' to call the RRT. In summary, the RN made three types of decisions: thought, enacted and operational.

Other key findings from the study concerned decision-making authority by the RRT RN in relation to rescuing. RRT RNs verbalised that during RRT training, they were told by their nursing leaders that they would be in the position to make decisions based on role authority because these leaders had confidence in their abilities to make the correct decisions to prevent adverse events. From the RRT RN perspective, they were given the message they were empowered to make decisions and that their decisions would be respected.

However, this was not always the case. For example, as an RRT intervention progressed, the RRT RN might make the thought decision that a patient needed to be transferred to a higher level of care. A key informant articulated that sometimes this decision was not based on standard patient data, such as vital signs, but rather it could be based on something more unusual, such as 'the patient is panicky' even though the patient had perfectly normal vital signs and skin colour. According to the informant, however, the patient being panicky was not sufficient data for the patient's physician to agree to transfer the patient to ICU. The RN reported that she imagined the physician thought she was an 'idiot' and a 'witch doctor of sorts' as she tried to explain that in her experience, this patient would need to be on a ventilator in a matter of a few hours. In this case, the enacted decision was made by the physician. Not convinced that the patient needed to be transferred, the enacted decision to not transfer the patient only delayed the ultimate transfer.

Findings support the idea that RNs are involved in decisionmaking and that decision-making was part of the rescuing process. The decision to take action by calling the RRT and transferring the patient to a higher level of care are two examples of RN decisions. The RRT protocols and consulting with others facilitated $\mathrm{RN}$ decision-making. The decision to transfer was facilitated by agreement among those involved when it was clear that intensive care was indicated as might be the case when a patient needed ventilator support or invasive monitoring. The RRT intervention was not always smooth in terms of RN decision-making; minor themes of conflicts and barriers emerged. While there were different types of decisions, thought decisions, enacted decisions and operational decisions, different members were empowered to make those decisions.

\section{RN-to-RN role synergy}

The rescuing process, carried out by RNs engaged in RRT interventions, involved role synergy characterised by $\mathrm{RN}-\mathrm{RN}$ consultation. Bedside RNs brought patient information to the situation. In some instances, a standardised communication format was used to describe the situation, background, assessment and recommendation. ${ }^{22}$ One bedside RN perceived her role in contributing to this consultation process as: 'I stay in the room the whole time...My role was to fill the team in on what was happening with the patient, the history, initial complaints, my assessment...they didn't really know the patient.'

The RRT RN contributed critical care knowledge and skills to the consultation and established clinical mastery in critical situations. Skills included: rapidly assessing, monitoring and changing the oxygen delivery method; initiating and managing intravenous fluids; and assessing potential need for intubation and communication. An RRT RN described the two-way interactive dialogue as: 'I used her [the bedside RN] as a resource to get the information. She used me as a resource also to figure out what was going on...we did have a dialogue.'

The interchange between the two RNs created the opportunity for focused questioning, an informed assessment of the patient led by the bedside RN, application of critical situation skills by the RRT RN and the opportunity for support and coaching. When the skill sets of the two RNs were combined, the RNs worked collaboratively to prevent further deterioration of the patient's clinical status and to prevent an adverse event. As one bedside RN described it: 'If a RRT is called, you have all 
the resources that you possibly need at the bedside so that the situation doesn't turn into a code arrest.'

The RN-RN consultation was a source of role synergy. Role synergy is the increased gain achieved from interaction and cooperative focus on the needs and urgency of the circumstance that is greater than achieved through individual efforts. The RRT RN augmented, reinforced and supported the bedside nurse. The RRT RNs' clinical expertise supplemented and supported the bedside $\mathrm{RN}$ to respond to a changing clinical situation and a patient at risk. Role synergy emerged from the bedside RNs need for support to interpret vague or ambiguous clinical signs and symptoms. RRT RNs contributed expert clinical judgement by calling on experience with critically ill patients, their knowledge of risks and the signs and symptoms of early clinical instability. This interchange was a collaborative sharing of relevant information to address their mutual goal of responding to changing patient needs. $\mathrm{RN}-\mathrm{RN}$ role synergy was a value-added contribution to preventing adverse events, which, in the case of an RRT intervention, is preventing a code arrest and is therefore an important contribution to patient safety by RNs.

\section{DISCUSSION}

Findings from $\mathrm{RNs}$ in acute care hospitals regarding their involvement and contribution to quality and patient safety through RRTs provide two important contributions that inform our understanding of how RNs engage in rescuing. The first contribution is a description of the decision-making process $\mathrm{RNs}$ use in rescuing patients. Bedside RNs use their knowledge of the RRT trigger protocol to make a thought decision to call the RRT. They use this approach to decide on the appropriate action to take to get additional support and make known the need for help, to get additional resources or to get urgent assistance to prevent an adverse event. In some cases, the protocol facilitates the decision process, as it provides a guiding structure for the RN to evaluate and interpret situational information and provides a context for making the enacting decision to call the RRT. The protocol frames the thought decision and supports the enactment decision by serving as a sanctioned rationale for the action of calling the RRT.

In contrast to the enabling structure of the RRT protocol, RNs encounter some individual-level and system-level obstacles as they make decisions about patient care safety usually regarding what level of care is most appropriate for the changing patient needs. RRT RNs felt empowered to make decisions and believed their decisions would be respected. However, in some cases, RN decision-making processes were complicated during $\mathrm{RN}$ and physician encounters. In this way, some of the hierarchical processes and traditional structures in hospitals complicated the decision-making RRT RNs employ in rescuing patients, even when there was administrative support and recognition of the need for these nurses to make decisions to prevent adverse events.

The second contribution to our understanding of the role of RNs and their involvement in RRTs is a description of the collegiality and role support demonstrated between RNs. Through $\mathrm{RN}-\mathrm{RN}$ role synergy, there is complementary alignment of the bedside RN and RRT RN in rescuing. Bedside RNs need the support of RRT RNs armed with critical situation skills. RRT RNs, usually critical care RNs, with expert clinical knowledge provide these skills. The acquisition of this expert clinical knowledge is gained through clinical experience, day after day, over time that informs a nurse's judgement and elevates nursing practice to a higher level of expertise. ${ }^{23}$ The bedside RNs' exposure to critical situations is not likely to be sufficient to develop this level of expert clinical knowledge. However, it is exactly at these times that bedside RNs need expert nursing consultation and support at the point of care. The support provided by the RRT RN was primarily a skillbased approach to assessment, clinical decision-making, communication and management of care emanating from knowledge and mastery of critical clinical situations. This RNto-RN role synergy likely empowered both $\mathrm{RNs}$ to take the best possible approach to care. Additionally, having RN-to-RN respect and collaboration in responding to the patient's critical needs was supportive and rewarding for both nurses. This meaningful exchange in the context of providing quality care may have vital significance in how all nurses feel about the unit and organisational environment in which they work and about their contribution to meeting the patient's needs.

\section{CONCLUSIONS}

Bedside RNs find the RRT supportive of their recognition that the patient is at risk, or care needs are changing. They benefit from having an RRTavailable because it is a formalised mechanism that brings needed resources right away. The process $\mathrm{RNs}$ use to make and enact decisions is complex and involves their clinical expertise, role relationships with physicians and traditional organisational structures. Their decision-making process can be inhibited by these interactions and traditional relationships.

The interactions between the RRT RNs and bedside RNs are an important aspect of understanding how the RRT adds value to the process of rescuing. Caregiver interactions such as the $\mathrm{RN}-\mathrm{RN}$ dialogue, support and consultation can contribute to the reliability and safety of the care delivered. The RRT RNs in this study were making valuable contributions to patient safety through the knowledge and skill they employed when rescuing patients in non-ICU patient care settings and through the role support they provided to bedside RNs. Thus, the nursing care provided through rescuing in the context of the RRT can make a critical difference in how a patient experiences an illness or change in health status.

Funding Funding provided by the Gordon and Betty Irene Moore Foundation. The funding source had no involvement, and the authors' work is independent of the funders. The funding was provided to the Association of California Nurse Leaders who served as the sponsor of the study. The sponsor had no role in the study design, collection, analysis or interpretation of data, writing the report or in the decision to submit the article for publication.

\section{Competing interests None.}

Ethics approval Ethics approval was provided by each hospital site IRB and UCLA IRB.

Contributors LSL is the guarantor with full responsibility for the work, conducted the study, had access to data and controlled the decision to publish.

Provenance and peer review Not commissioned; externally peer reviewed.

\section{REFERENCES}

1. Berwick DM, Calkins DR, McCannon CJ, et al. The $100 \mathrm{~K}$ lives campaign: setting a goal and a deadline for improving health care. JAMA 2006;295:324-7.

2. Institute of Medicine. Transforming the work environment of nurses: keeping patients safe. Washington, D.C.: National Academies Press, 2004.

3. Bellomo R, Goldsmith D, Uchino S, et al. Prospective controlled trial of effect of an emergency team on postoperative morbidity and mortality rates. Crit Care Med 2004;32:916-21

4. Bristow PJ, Hillman KM, Chey T, et al. Rates of in-hospital arrests, deaths and intensive care admissions: the effect of a medical emergency team. Med J Aust 2000;173:236-40.

5. Buist MD, Moore GE, Bernard SA, et al. Effects of a medical emergency team on reduction of incidence of and mortality from unexpected cardiac arrests in hospital: preliminary study. BMJ 2002;324:387-90.

6. DeVita MA, Braithwaite RS, Mahidhara R, et al. Use of medical emergency team responses to reduce hospital cardiopulmonary arrests. Qual Saf Health Care 2004;13:251-4. 
7. Institute for Healthcare Improvement [homepage on the Internet]. Rapid response teams, 2005. http://www.hi.org/lH//Programs/Campaign/Campaign.htm. cited 2007 February 5.

8. Hillman K, Chen J, Cretikos M, et al. MERIT study investigators. Introduction of the medical emergency team (MET) system: a cluster-randomized controlled trial. Lancet 2005; $365: 2091-7$

9. Winters BD, Pham J, Provonost PJ. Rapid response teams-walk, don't run. JAMA 2006;296:1645-7.

10. Galhotra S, DeVita MA, Simmons RL, et al, MERIT Committee. Mature rapid response system and potentially avoidable cardiopulmonary arrests in hospital. Qual Saf Health Care 2007;16:260-5.

11. McCloskey J, Bulechek G, eds. Nursing interventions classifications (NIC). 3rd edn. St Louis (M0): Mosby, 2000. p. 629.

12. National Quality Forum. National voluntary consensus standards for nursingsensitive care: an initial performance measure set. A consensus report. Washington, D.C.: National Quality Forum, 2004

13. Tourangeau AE, Doran DM, Hall LM, et al. Impact of hospital nursing care on 30-day mortality for acute medical patients. J Adv Nurs 2006; 57:32-44.

14. Aiken L, Clarke SP, Sloane DM, et al. Hospital nurse staffing and patient mortality, nurse burnout, and job dissatisfaction. JAMA 2002;288:1987-93.
15. Aiken L, Clarke SP, Cheung RB, et al. Educational levels of hospital nurses and surgical patient mortality. JAMA 2003;290:1617-23.

16. Needleman J, Buerhaus P, Mattke $S$, et al. Nurse-staffing levels and the quality of care in hospitals. N Engl J Med 2002;364:1715-22.

17. Clarke SP. Failure to rescue: lessons from missed care opportunities. Nurs Inq 2002;11:67-71.

18. Clarke SP, Aiken LH. Failure to rescue: needless deaths are prime examples of the need for more nurses at the bedside. Am J Nurs 2003;10:42-7.

19. Cretikos M, Bellomo R. Overview of various medical emergency team models, In: DeVita Michael, Hillman, Kenneth, Bellomo, Rinaldo, eds. Medical emergency teams: implementation and outcome measurement. New York: Springer, 2006:110.

20. 5 Million Lives Campaign. Getting starter kit: rapid response teams. Cambridge (MA): Institute for Healthcare Improvement, 2008. http:///www.ihi.org. Cited 2009 April 10.

21. Teekman B. Exploring reflective thinking in nursing practice. J Adv Nurs 2000;31:1125-35.

22. Leonard M, Graham S, Taggart B. The human factor: effective teamwork and communication in patient strategy. In: Leonard Michael, Frankel Alan, Simmonds Terri, et al, eds. Achieving safe and reliable healthcare: strategies and solutions. Chicago (IL): Health Administration Press, 2004. p. 60.

23. Benner P. From novice to expert: excellence and power in clinical nursing practice commemorative edition. Upper Saddle River (NJ): Prentice Hall, 2000. 